\title{
Flt1 acts as a negative regulator of tip cell formation and branching morphogenesis in the zebrafish embryo
}

\author{
Janna Krueger ${ }^{1,2}$, Dong Liu ${ }^{1}$, Katja Scholz ${ }^{1,3}$, Anja Zimmer ${ }^{1,3}$, Yu Shi ${ }^{1,3}$, Christian Klein ${ }^{1}$, Arndt Siekmann ${ }^{4}$, \\ Stefan Schulte-Merker ${ }^{5}$, Melissa Cudmore ${ }^{6}$, Asif Ahmed ${ }^{6}$ and Ferdinand le Noble ${ }^{1,2,3, *}$
}

\begin{abstract}
SUMMARY
Endothelial tip cells guide angiogenic sprouts by exploring the local environment for guidance cues such as vascular endothelial growth factor (VegfA). Here we present Flt1 (Vegf receptor 1) loss- and gain-of-function data in zebrafish showing that Flt1 regulates tip cell formation and arterial branching morphogenesis. Zebrafish embryos expressed soluble Flt1 (sFlt1) and membrane-bound Flt1 (mFlt1). In $T g\left(f l t 1^{B A C}: y f p\right) \times T g(k d r l: r a s-c h e r r y)^{s 916}$ embryos, flt1:yfp was expressed in tip, stalk and base cells of segmental artery sprouts and overlapped with $k d r l$ :cherry expression in these domains. flt 1 morphants showed increased tip cell numbers, enhanced angiogenic behavior and hyperbranching of segmental artery sprouts. The additional arterial branches developed into functional vessels carrying blood flow. In support of a functional role for the extracellular VEGF-binding domain of Flt1, overexpression of sflt1 or mflt1 rescued aberrant branching in flt 1 morphants, and overexpression of sflt1 or mflt 1 in controls resulted in short arterial sprouts with reduced numbers of filopodia. flt 1 morphants showed reduced expression of Notch receptors and of the Notch downstream target efnb2a, and ectopic expression of flt4 in arteries, consistent with loss of Notch signaling. Conditional overexpression of the notch1a intracellular cleaved domain in flt1 morphants restored segmental artery patterning. The developing nervous system of the trunk contributed to the distribution of Flt 1 , and the loss of flt 1 affected neurons. Thus, Flt1 acts in a Notch-dependent manner as a negative regulator of tip cell differentiation and branching. Flt1 distribution may be fine-tuned, involving interactions with the developing nervous system.
\end{abstract}

KEY WORDS: FIt1, VEGF, Angiogenesis, Notch, Tip cells, Nerves, Zebrafish

\section{INTRODUCTION}

Sprouting angiogenesis plays a crucial role in vascular network expansion during normal development and pathophysiological conditions (Carmeliet et al., 2009). Angiogenic sprouting involves the specification of endothelial cells into tip and stalk cells in the growing sprout (Phng and Gerhardt, 2009), with the tip cell being localized at the leading edge of the sprout. Tip cells extend numerous filopodia and respond to guidance cues including vascular endothelial growth factor (VegfA), directing the movement of the sprout. The endothelial cells that follow the tip cells are called stalk cells. Stalk cells have fewer filopodia, they proliferate in response to VegfA, and they contribute to vessel lumen formation. Within the sprout, endothelial cells dynamically compete with each other for the tip cell position (Jakobsson et al., 2010). The full repertoire of genes controlling endothelial tip cell differentiation is unknown, but VEGF receptors including Flk1 (Kdr, Vegfr2) and Flt4 (Vegfr3) and Notch-delta-like 4 (Dll4) signaling pathways play key roles (Benedito et al., 2009; Gerhardt et al., 2003; Hellstrom et al., 2007; Lobov et al., 2007; Siekmann

\footnotetext{
${ }^{1}$ Department of Angiogenesis and Cardiovascular Pathology, Max-Delbrueck Center for Molecular Medicine, D13125 Berlin, Germany. ${ }^{2}$ Center for Stroke Research Berlin, Charite, D10117 Berlin, Germany. ${ }^{3}$ Experimental and Clinical Research Center of the Charite and the Max-Delbrueck Center for Molecular Medicine, D13125 Berlin, Germany. ${ }^{4}$ Max-Planck Institute for Molecular Biomedicine, D48149 Muenster, Germany. ${ }^{5}$ Hubrecht Institute-KNAW and UMC, 3584CT Utrecht, The Netherlands. ${ }^{6}$ UoE/BHF Centre for Cardiovascular Science, University of Edinburgh, Edinburgh EH16 4TJ, UK.
}

*Author for correspondence (lenoble@mdc-berlin.de)

Accepted 24 February 2011 and Lawson, 2007; Suchting et al., 2007; Tammela et al., 2008). Here, we present genetic evidence that flt1 (vegfrl) acts, in a Notch-dependent manner, as a negative regulator of tip cell formation and sprout guidance in the trunk vasculature of zebrafish embryos.

In mammals, thus far three VEGF receptors have been identified, namely Flt1, Flk1 and Flt4. In all other vertebrate classes, four receptors exist (Bussmann et al., 2008). These receptors bind VEGF ligands and regulate angiogenesis and lymphangiogenesis (Alitalo et al., 2005; Ferrara et al., 2003). Zebrafish VEGF receptor proteins are encoded by the $f t t 1, k d r$ (previously $k d r b), k d r$-like ( $k d r l$, previously $k d r a$ ) and the flt4 (Bussmann et al., 2008) orthologs. Whereas the roles of $f l k 1, k d r$ and $k d r l$, and of $f l t 4$ in mediating vascular development in zebrafish are well described (Covassin et al., 2009; Covassin et al., 2006; Habeck et al., 2002; Hogan et al., 2009b; Lawson et al., 2002; Liao et al., 1997; Siekmann and Lawson, 2007), surprisingly little is known about flt1. Thus far, flt 1 has been implicated in the regulation of embryonic heart function and cardiac morphogenesis (Bussmann et al., 2007; Rottbauer et al., 2005), but its specific role in endothelial tip cell formation in zebrafish is unknown.

In mammals, Flt1 encodes one membrane-bound form, which has an intracellular tyrosine kinase signaling domain, and soluble forms, which only contain the extracellular VEGF-binding domain (Sela et al., 2008). Genetic studies in mice have shown that Flt1 plays a pivotal role in both developmental and pathological forms of angiogenesis (Ferrara et al., 2003; Fong et al., 1995). Flt1-null mice die at $\sim$ E8.5 and show disorganized blood vessels and overgrowth of endothelial cells (Fong et al., 1995). However, Flt1 tyrosine kinase-deficient homozygous mice $\left(F l t 1^{T K-/-}\right)$ survive and appear to develop normal vessels, suggesting that the tyrosine 
kinase activity is dispensable for vascular morphogenesis (Hiratsuka et al., 1998). Other studies show that the soluble form of Flt1 regulates vessel development by acting as a sink for VegfA, thus limiting both extracellular VegfA availability and signaling through Flk1 (Ambati et al., 2006; Gerhardt et al., 2003; Roberts et al., 2004).

Here, we focused on the role of flt 1 in tip cell formation and branching morphogenesis of segmental vessels in the trunk of zebrafish embryos. We show that flt 1 regulates tip cell formation and segmental artery branching. We furthermore reveal a contribution of the developing nervous system to the distribution of Flt1.

\section{MATERIALS AND METHODS}

\section{Zebrafish experiments}

Zebrafish were kept at $26.5^{\circ} \mathrm{C}$ and bred under standard conditions $\operatorname{Tg}(\text { fli:egfp })^{y 1}, \quad T g(k d r l: h r a s-m c h e r r y)^{s 896}, \quad T g(k d r l: r a s \text {-cherry })^{s 916}$, $\operatorname{Tg}\left(f l t 1^{B A C}: y f p\right), \operatorname{Tg}($ huC:egfp), $\operatorname{Tg}(h s p 70:$ Gal4) and $\operatorname{Tg}$ (uas:notchla-ICD) transgenic zebrafish lines were used as described (Chi et al., 2008; Hogan et al., 2009a; Isogai et al., 2003; Lawson and Weinstein, 2002; Park et al., 2000; Siekmann and Lawson, 2007). Morpholino antisense oligomers (MOs; Gene Tools) were prepared at a stock concentration of $1 \mathrm{mM}$ according to the manufacturer's protocol. MOs were injected into the yolk of one-cell stage embryos. We used two MOs targeting zebrafish flt 1 : ATGblocking MO, 5'-ATATCGAACATTCTCTTGGTCTTGC-3' (Flt1MO, $3 \mathrm{ng}$ ); and a 5'UTR-targeting MO, 5'-CTTGAGGGTGTTTGTTTGGAGATGA-3' (5'UTR-MO, $1.5 \mathrm{ng})$. dll4 splicing MO (5'-TAGGGTTTAGTCTTACCTTGGTCAC-3') and the pul MO (5'-GATATACTGATACTCCATTGGTGGT-3') were used as described (Hsu et al., 2004; Leslie et al., 2007). The standard control MO was 5'-CTCTTACCTCAGTTACAATTTATA-3' (control MO, 3 ng).

Overexpression of Notch was performed using $\operatorname{Tg}($ hsp 70 :Gal4) $\times$ $T g$ (uas:notchla-ICD) double-transgenic embryos. Embryos were heat shocked at $40^{\circ} \mathrm{C}$ for 30 minutes at the $16-18$ somite stage and then incubated at $26.5^{\circ} \mathrm{C}$ for further experiments.

\section{Isolation and cloning of sFlt1}

We identified the $3^{\prime}$ and $5^{\prime}$ ends of the sflt 1 isoform by RACE PCR using Clontech RACE Kits on first-strand cDNA prepared from RNA of zebrafish embryos (30 hpf). The primers for the $3^{\prime}$ and $5^{\prime}$ RACE are listed in Table S1 in the supplementary material. PCR fragments were cloned into the pGEM-T Easy vector (Promega) and sequenced. The sFlt1 sequence has GenBank Accession Number JF330410.1.

\section{Expression constructs}

For rescue experiments, poly(A)-capped mRNA was injected into one-cell stage embryos. Zebrafish $m$ flt 1 (GenBank accession 72535147) and sflt 1 were cloned into the PCS2+ vector (S. Seyfried, MDC, Berlin, Germany). Sense-capped mRNA was generated by SP6 Message mMachine (Ambion) and, after purification, $2 \mathrm{nl}$ was injected at $50 \mathrm{ng} / \mu \mathrm{l}$. The Gateway cloning system was used in accordance with the manufacturer's instructions (Invitrogen) to express sflt 1 under the control of the fli promoter. For the $\mathrm{BP}$ reaction, the amplified $s f l t 1$ product was cloned into $\mathrm{pCR} / \mathrm{GW} / \mathrm{TOPO}$ (Invitrogen). The subsequent LR reaction was performed with pTolfliep:cherryDest (Villefranc et al., 2007). To express sflt1 under the control of the $k d r l$ promoter, egfp-sfltl was inserted into pminiTolkdrl. The final constructs, pTolfliep:cherry-sflt1 and pminiTolkdrl:egfp-sflt1, were injected along with Tol2 transposase RNA into one-cell stage embryos.

\section{Gene expression analysis by Taqman}

Total RNA of zebrafish was isolated with TRIzol (Invitrogen) and purified using the RNeasy Mini Kit (Qiagen). The quantity and quality of extracted RNA were measured using an Agilent 2100 Bioanalyzer (Agilent Technologies, Palo Alto, CA, USA) according to the manufacturer's instructions, followed by cDNA synthesis using the Thermoscript First-Strand Synthesis System (Invitrogen). Primer probe sets (FAM and TAMRA labels) were obtained from BioTez (Berlin,
Germany); sequences are shown in Table S1 in the supplementary material. Amplification was carried out using an ABI Prism 7000 thermocycler (Applied Biosystems). Gene expression data were normalized against elongation factor 1-alpha.

\section{Whole-mount in situ hybridization, western blot and} immunohistochemistry

Whole-mount in situ hybridization with antisense RNA probes was performed as described (Jowett and Lettice, 1994); see Table S1 in the supplementary material for details. The polyclonal antibody against zebrafish Flt1 extracellular domain (CQVTSGPSKRETNTT; Eurogentec, Belgium) was purified by affinity matrix and used at 1:1000. To verify the specificity of the Flt1 antibody in a western blot, we overexpressed zebrafish Flt1 coupled to GFP. Immunoprecipitation was performed on extracts from HeLa cells transfected with the expression vector pEGFPC3-zebrafish $m$ flt 1 . At 24 hours after transfection, the fusion protein was purified from cell lysates using an anti-GFP antibody (Sigma-Aldrich) and protein G sepharose (Invitrogen).

For immunofluorescence staining, embryos were fixed in $4 \%$ paraformaldehyde and permeabilized with methanol and acetone. To detect Flt1 staining in vessels, the immune signal was amplified by tyramide signal amplification (TSA; Perkin Elmer). Anti-phospho-histone H3 (Ser10, Millipore), ImmunoPure goat anti-rabbit IgG (Thermo scientific) and zn-12 (ZFIN) antibodies were used. As secondary antibodies, polyclonal goat anti-rabbit HRP (Dako, Denmark) and Alexa Fluor 633 goat anti-rabbit IgG (Invitrogen) were used.

\section{Drug treatment}

DAPT was purchased from Sigma-Aldrich and dissolved in DMSO. DAPT was used at a final concentration of $100 \mu \mathrm{M}$ diluted as described (Leslie et al., 2007). Egg water containing DMSO alone was utilized as control.

\section{Imaging}

In vivo blood flow was imaged using a Zeiss intravital microscopy setup (Zeiss Axioscope A1, Carl Zeiss MicroImaging, Jena, Germany) with a $20 \times($ N.A. $=0.50)$ water-emersion objective. Images were stored on digital tape (Sony DVCAM64) using a digital video recorder (Sony DVCAMDSR-25). For confocal time-lapse imaging of segmental vessel sprouting in $\operatorname{Tg}(f l i: e g f p)^{y l}$ and $\operatorname{Tg}(k d r l: h r a s-m c h e r r y)^{s 896}$ zebrafish, embryos were injected with $f l t 1$ or control MO, anesthetized with egg water $/ 0.16 \mathrm{mg} / \mathrm{ml}$ tricaine/1\% 1-phenyl-2-thiourea (Sigma) and embedded in 1\% agarose. Using a $25 \times$ objective, confocal stack images of the trunk region were obtained in time intervals of 20 minutes. Confocal imaging was performed with a Zeiss LSM 510 microscope (Carl Zeiss MicroImaging). The analysis was performed using Zeiss ZEN software.

\section{Statistics}

Data are expressed as mean \pm s.e.m. Statistical analysis was performed using Student's two-tailed $t$-test $(P<0.05)$.

\section{RESULTS \\ Zebrafish embryos express membrane-bound and soluble Flt1}

We examined the expression pattern of flt 1 isoforms in zebrafish embryos by Taqman analysis, western blot and whole-mount in situ hybridization (Fig. 1; see Fig. S1 in the supplementary material). We detected one long form of flt 1 compatible with membranebound Flt1 (mFlt1) (Fig. 1A; see Fig. S1A-C in the supplementary material), and by $3^{\prime}$ and $5^{\prime}$ RACE-PCR we identified a shorter form of flt1 compatible with soluble Flt1 (sFlt1) (Fig. 1A; see Fig. $\mathrm{S} 1 \mathrm{~A}-\mathrm{C}$ in the supplementary material). The sflt1 isoform resulted from alternative splicing, causing an extension of exon 10. This extension encodes the $5^{\prime}$ region of intron 10 . Based on this mRNA sequence in sflt1 (Fig. 1A; see Fig. S1C in the supplementary material) that is not shared by $m f l t 1$, we were able to design isoform-specific Taqman primers and antisense riboprobes for in situ hybridization (see Table S1 in the supplementary material). 
A

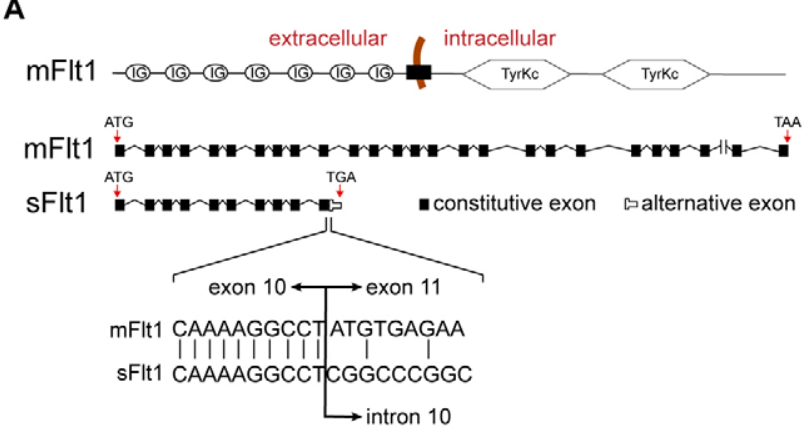

C

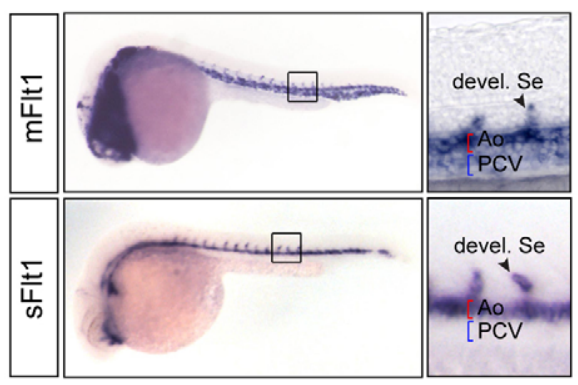

B

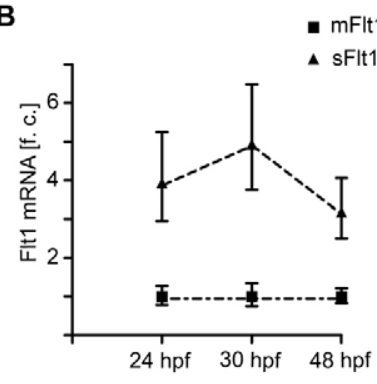

Fig. 1. Expression of flt 1 isoforms in zebrafish

embryos. (A) Structural organization of membrane-bound flt1 ( $m f l t 1$ ) and soluble flt1 (sflt 1). sflt1 contains a unique mRNA sequence, not present in $m f l t 1$, that encodes the $5^{\prime}$ region of intron 10. (B) Taqman analysis shows higher expression of sflt 1 than mflt 1 in control embryos at $24 \mathrm{hpf}$, $30 \mathrm{hpf}$ and $48 \mathrm{hpf}$. f.c., fold change relative to $\mathrm{mflt} 1$ expression at indicated time-points. Error bars indicate s.e.m. (200 embryos/group from four separate experiments). (C) Whole-mount in situ hybridization with antisense riboprobes directed against $m f l t 1$ or sflt1; embryos at $30 \mathrm{hpf}$. Note that $\mathrm{mflt} 1$ is expressed in segmental sprouts, aorta and cardinal vein; sflt 1 is expressed in segmental sprouts and aorta. Ao, dorsal aorta; PCV, posterior cardinal vein; Se, segmental artery.
Taqman analysis revealed $\sim 3$ - to 5-fold higher expression levels of sflt1 compared with mflt1 during the period 24-48 hours postfertilization (hpf) (Fig. 1B). In toto in situ hybridization showed that mflt1 was expressed in the aorta, the cardinal vein and developing segmental vessels (Fig. 1C). Expression of $s f l t 1$ was detectable in the aorta and segmental vessels, but not in the posterior cardinal vein (Fig. 1C). Expression of mFlt1 and sFlt1 protein in control embryos was confirmed by western blot (Fig. 2A) using whole-embryo membrane fractions and lysate, respectively.

\section{Flt1 regulates segmental vessel branching morphogenesis}

flt1 was knocked down by injecting an ATG-targeting MO into $T g(f l i 1: e G F P)^{y 1}$ zebrafish embryos (Fig. 2). For technical reasons, during imaging embryos were incubated at $26.5^{\circ} \mathrm{C}$. Knockdown of flt1 using an ATG-targeting MO reduced mFlt1 and sFlt1 protein levels (Fig. 2A) and resulted in excessive branching of segmental vessels (Fig. 2B). Similar branching defects were observed using the flt1 5'UTR-targeting MO (see Fig. S2E in the supplementary material). For the ATG-blocking MO, effects were observed at a dose of $3 \mathrm{ng}$; at higher dosages, we observed dysmorphogenesis of the embryo, and these were therefore not examined in detail. We observed striking changes in segmental vessel branching of flt 1 morphants (Fig. 2B). Although the position of the initial sprout formation was not affected (Fig. 2B, $30 \mathrm{hpf}$ ), at later stages segmental vessels formed aberrant connections laterally, both in the posterior and anterior direction, connecting with the segmental vessels of the adjacent somites (Fig. 2B, 48 and $72 \mathrm{hpf}$ ). Once the intersegmental vessel (ISV) sprouts reached the horizontal myoseptum, they subsequently deviated by an angle of $~ 20-30$ degrees in the anterior or posterior direction. The progression of the sprouts appeared slower in flt1 morphants than in the control. The aberrant vessel connections formed a lumen, carried blood flow and were not pruned (Fig. 2B, 72 hpf; see Movies 1 and 2 in the supplementary material). This aberrant branching phenotype was observed in $75 \%$ of all $f l t 1$ ATG-blocking MO-injected animals (Fig. 2D; $n=420$, six separate sets of experiments), but never in controls ( $n=420$, six separate sets of experiments).

To verify that the vascular phenotype was due to a reduction in flt 1 expression, we performed rescue experiments by co-injection of the flt 1 ATG-targeting MO with either $m f l t 1$ or sflt 1 mRNA (Fig. 2C). Co-injection of flt $1 \mathrm{MO}$ with $m$ flt 1 or sflt 1 mRNA resulted in a significant rescue of the vascular phenotype (Fig. 2C,D).

Consistent with a role for flt1 in ISV branching, injection of mflt1 or sflt 1 mRNA increased Flt1 protein levels (see Fig. S2A in the supplementary material) and reduced sprouting of the segmental arteries (Fig. 2E,F). Although the initial segmental sprout positioning was correct, sprout expansion stopped halfway towards the dorsal roof. The tip cells of such sprouts displayed shorter or no filopodial extensions (Fig. 2E).

flt 1 has been implicated in the regulation of heart rate (Rottbauer et al., 2005). We assessed heart rate (see Fig. S2C,D in the supplementary material) and perfusion of the trunk vasculature using an intravital imaging approach (see Movies 3 and 4 in the supplementary material). Heart rate was lower in flt 1 morphants than in control MO-injected embryos, but dorsal aorta, posterior cardinal vein and segmental vessels were perfused with blood (see Movies 3 and 4 in the supplementary material), indicating that a circulatory loop was established in flt 1 morphants.

\section{Flt1 regulates tip cell differentiation}

We imaged segmental artery sprouting in flt 1 morphants and observed an increase in tip cell number and angiogenic sprouting behavior (Fig. 3; see Fig. S3A and Movies 5 and 6 in the supplementary material). In control embryos, segmental sprouts consisted of three endothelial cells arranged as follows: tip cell, followed by the connecter/stalk cell, followed by the base cell, the latter connecting to the aorta (Fig. 3A; see Movie 5 in the supplementary material). In segmental vessels of flt 1 morphants, we observed increased endothelial cell numbers and more cells with endothelial tip cell characteristics (Fig. 3A-D; see Movie 6 
A WB: Flt1

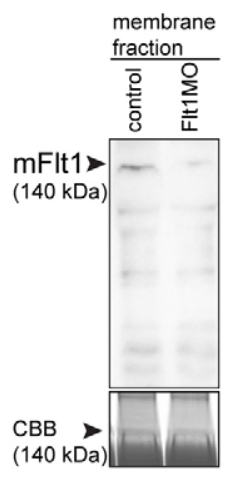

C

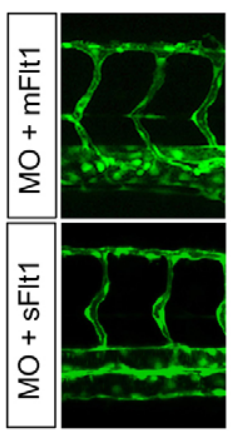

B
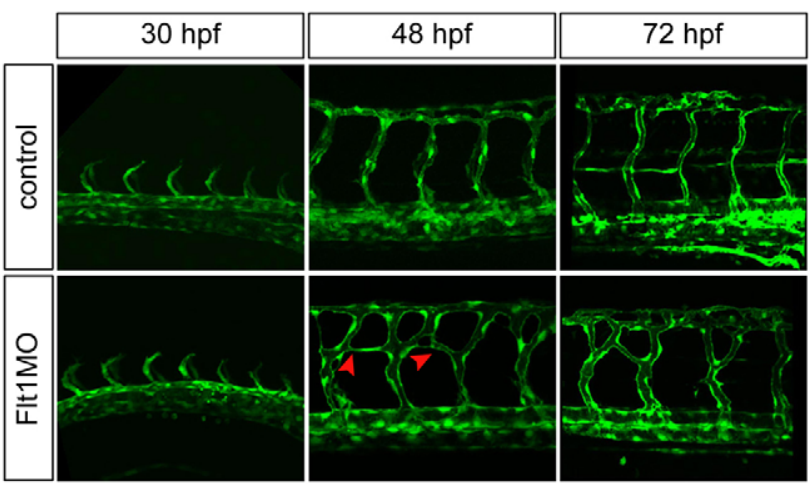

E

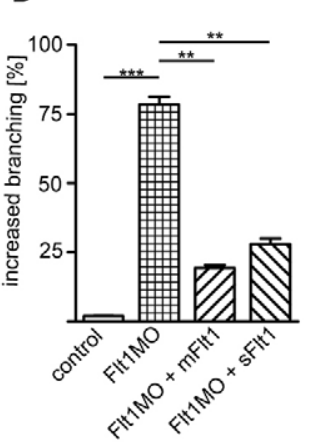

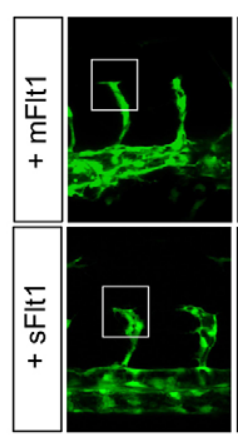

F

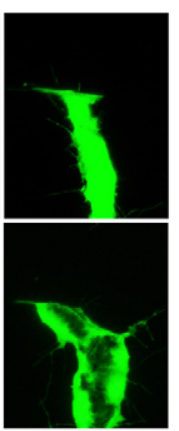

Fig. 2. Flt1 regulates segmental vessel branching morphogenesis. $(\mathbf{A}, \mathbf{B})$ Reduced mFlt1 and sFlt1 protein levels (A) and excessive segmental vessel branching (B) in zebrafish flt1 morphants. The red arrowheads in B indicate the aberrant connection between adjacent segmental vessels. (C,D) Injection of mRNA encoding mflt1 or sflt1 rescued segmental branching defects in flt1 morphants. $\mathrm{MO}$, flt1 ATG morpholino. $y$-axis shows percentage of examined embryos with aberant segmental vessel branching, as shown in $B$ (lower middle panel; 120 embryos/ group from four separate

experiments). (E,F) Overexpression of mflt1 (top row) or sflt1 (bottom row) in control embryos results in short segmental artery sprouts ( $E$, left) and reduced filopodia extensions ( $E$, right). The boxed regions are shown at higher magnification in the righthand panels. $y$-axis shows

percentage of embryos with reduced segmental vessel sprouts, as shown in $\mathrm{E} .{ }^{*}, P<0.01 ; * * *, P<0.001$; Student's t-test. Error bars indicate s.e.m. (120 embryos/group from three separate experiments). in the supplementary material). Specifically, flt1 morphants showed: (1) segmental vessels spearheaded by two endothelial cells displaying filopodia extensions; (2) increased filopodia extensions throughout the segmental sprout; (3) endothelial cells at a position normally taken by the connector/stalk or base cells that displayed filopodia extensions and formed connections to adjacent vessels; (4) increased endothelial cell numbers in segmental vessels (Fig. 3A-D; see Movies 5 and 6 in the supplementary material). Dorsal extension of ISVs in flt 1 morphants appeared slower than in controls, which might be due to altered proliferation rates.

In the cell-tracking experiment, flt1 morphant tip cells were identified that gave rise to two progeny cells, both of which displayed filopodia extensions and a tip cell phenotype (Fig. 3A; see Fig. S3A in the supplementary material). In addition, endothelial cells of flt 1 morphants were highly motile and frequently moved away from the base of the segmental vessel to then integrate at the leading edge, adjacent to the tip cell that was already present in this area (see Movie 6 in the supplementary material). At the leading edge, these endothelial cells extended filopodia, resulting in a segmental vessel spearheaded by two endothelial tip cells. Moreover, between the sprouting front and the connection with the aorta, additional endothelial cells displaying filopodia extensions were observed (see Movie 6 in the supplementary material). These endothelial cells attempted to make connections with adjacent segmental vessels, and, at the level at which these connections occurred, lumen formation had commenced. Based on their anatomical location these cells may be assigned as connector/stalk or base cells. Based on their morphological features, filopodia extensions and formation of connections with other vessels, they may be assigned as tip cells.
Collectively, these results suggest that loss of flt 1 results in increased tip cell formation. The observed increase in angiogenic cell behavior and migration within the segmental sprout is reminiscent of the effects of loss of Notch signaling (Siekmann and Lawson, 2007).

\section{Tip and stalk cell marker expression and Notch signaling in flt1 morphants}

The Notch-Dll4 signaling pathway has been implicated in the regulation of tip and stalk cell differentiation (Phng and Gerhardt, 2009; Roca and Adams, 2007) and in angiogenic cell behavior in segmental vessels (Hogan et al., 2009b; Siekmann and Lawson, 2007). Expression of the tip cell markers $k d r l$, flt4 and dll4 and of the stalk cell markers nrarpa and nrarpb (Phng et al., 2009) was evaluated in flt 1 morphants (Fig. 4A,B). In $k d r l$ reporter fish, the leading edge of the segmental vessel is clearly wider than normal, compatible with the presence of more than one tip cell guiding the sprout. Similar results were obtained by $k d r l$ whole-mount in situ hybridization. Reduced expression of nrarpa and nrarpb in flt 1 morphants (Fig. 4B) suggests conversion of stalk/connector cells into tip cells. In support of this, and as described above, in flt 1 morphants, at the anatomical location normally occupied by stalk/connector or base cells, endothelial cells displaying filopodia extensions were observed (see Movie 6 in the supplementary material). Since some of these cells were in the process of creating a vessel lumen, a feature that is normally assigned to a stalk/connector cell, it could be argued that these cells have a mixed tip cell/stalk cell phenotype.

In flt 1 morphants, we observed ectopic flt 4 expression in the dorsal aorta (Fig. 4A), a feature previously associated with loss of Notch signaling (Siekmann and Lawson, 2007). Since dll4 
A

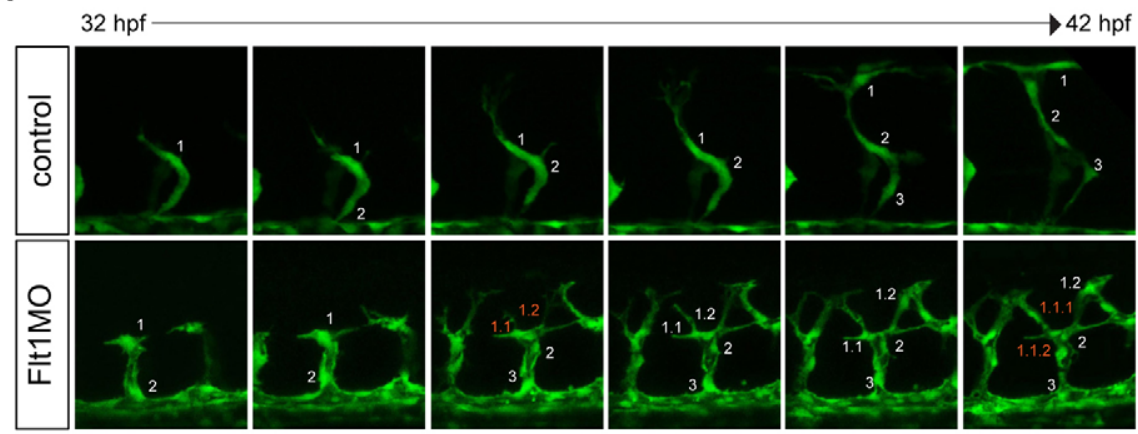

B

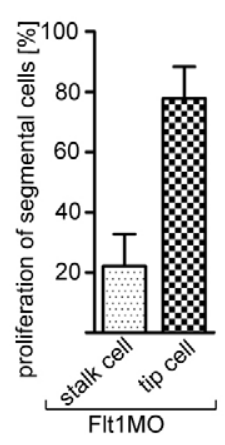

C

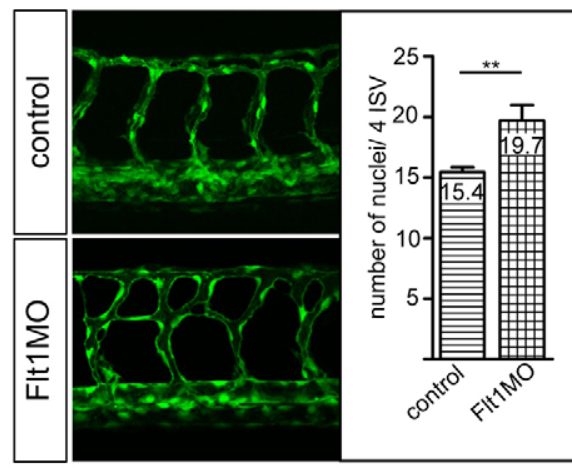

D

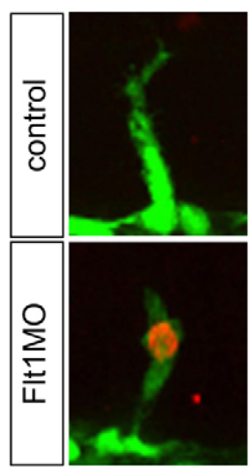

Fig. 3. Flt1 regulates endothelial tip cell formation. (A,B) Time-lapse in vivo imaging shows increased tip cell numbers and proliferating endothelial tip cells in segmental vessels of zebrafish flt1 morphants. Note that in flt 1 morphants the leading cell number 1 gives rise to two progeny cells termed 1.1 and 1.2 . $y$-axis shows the percentage of examined flt 1 morphants with proliferation in tip or stalk cells. (C) Quantification of endothelial cell numbers in four consecutive segmental vessel (ISV) - dorsal longitudinal anastomotic vessel (DLAV) loops reveals an increase in flt1 morphants. (D) Antiphospho-histone $\mathrm{H} 3$ staining confirms endothelial proliferation in segmental vessels of flt1 morphants. **, $P<0.01$; Student's $t$-test. Error bars indicate s.e.m. expression was maintained in flt1 morphants (Fig. 4A,B), we examined changes in Notch expression. Expression of the receptors notch $1 a$, notch $1 b$, notch 2 and notch3, the Notch ligands jagla, jag $1 b$ and jag2, and the Notch downstream target efnb2a was reduced in flt1 morphants (Fig. 4C,D). These data suggest that loss of Notch signaling in flt1 morphants most likely occurs through downregulation of Notch receptors.

In flt1 morphants, conditional overexpression of the notch1a intracellular cleaved domain (NICD), using $\operatorname{Tg}(h s p 70:$ Gal4 $) \times$ $\mathrm{Tg}$ (UAS:notchla-ICD) transgenic embryos as described (Siekmann and Lawson, 2007), inhibited excessive sprouting and restored segmental patterning (Fig. 4E; see Fig. S3B-F in the supplementary material). Overexpression of notch 1 a-ICD in control embryos resulted in severely reduced segmental vessel sprouting (see Fig. $\mathrm{S} 3 \mathrm{~B}, \mathrm{E}$ in the supplementary material). Expression of flt $1 \mathrm{mRNA}$ increased after conditional overexpression of notchla-ICD (see Fig. S3G in the supplementary material). When compared with untreated controls, in flt 1 morphants with overexpression of NICD, endothelial nuclei were more frequently found at a level below the horizontal myoseptum or at the base of the segmental vessel (Fig. $4 \mathrm{E}$; see Fig. S3F in the supplementary material). This suggests that restoring Notch activity affected endothelial cell movements in the sprout. However, segmental sprouts reached the dorsal roof and formed a dorsal longitudinal anastomotic vessel (DLAV), indicating that tip cell differentiation per se is not completely suppressed.

To test whether loss of Notch-D114 signaling is sufficient to explain the vascular branching pattern observed in flt1 morphants, we knocked down dll4 (Fig. 4F) or treated normal embryos with the Notch $\gamma$-secretase inhibitor DAPT (Fig. 4E). In line with previous reports (Leslie et al., 2007), both approaches resulted in endothelial sprouting phenotypes at the level of the DLAV (Fig. 4F, red arrows and arrowheads), but the segmental vessel branching pattern was not comparable to that of flt1 morphants (Fig. 4F, yellow arrowhead). Thus, loss of flt 1 is associated with loss of Notch, but loss of Notch signaling did not phenocopy that of flt 1 morphants.

\section{Distribution of Flt1 in vessels and the nervous system}

We examined the expression and distribution of Flt1 in more detail using $T g\left(f l t 1^{B A C}: y f p\right) \times T g(k d r l \text { :ras-cherry })^{s 916}$ double-transgenic embryos and Flt1 immunostaining. In $T g\left(f l t 1^{B A C}: y f p\right)$ embryos (Hogan et al., 2009a), YFP expression was mainly observed in the arterial domain including the aorta and the segmental arteries (Fig. 5A,B), with YFP expression in the venous domain being less pronounced. Detailed examination of segmental arterial vessel sprouts revealed YFP expression in tip cells and filopodia, stalk cells and base cells (Fig. 5A, arrowhead). In segmental sprouts, the expression domain of flt 1:yfp was comparable to that of kdrl:cherry (Fig. 5A). At $30 \mathrm{hpf}$, flt1:yfp expression was restricted to the vasculature; YFP expression in neural tissue was not observed at this time. By contrast, in $T g\left(f l t 1^{B A C}: y f p\right)$ embryos at $48 \mathrm{hpf}$, we also noted non-vascular YFP expression domains, most notably in the spinal cord neurons and their axonal projections (Fig. 5B, arrowhead).

We next knocked down flt1 using the ATG-blocking MO in $\operatorname{Tg}\left(f l t 1^{B A C}: y f p\right) \times \operatorname{Tg}(k d r l: \text { ras-cherry })^{s 916}$ double-transgenic embryos and observed hyperbranching of segmental arteries, similar to that observed in fli transgenics (Fig. 5B, left panels). The neuronal YFP expression domain was lost in flt1 morphants (Fig. 5B, lower left panel). In $T g(h u C: e g f p)$ neuronal reporter fish, knockdown of flt1 also affected neurons (Fig. 5B, right panels; see Fig. S4D in the supplementary material). This loss of neurons might also explain the reduced Notch receptor and ligand expression in the neural tube (Fig. 4C and Fig. 5B, right panels). 


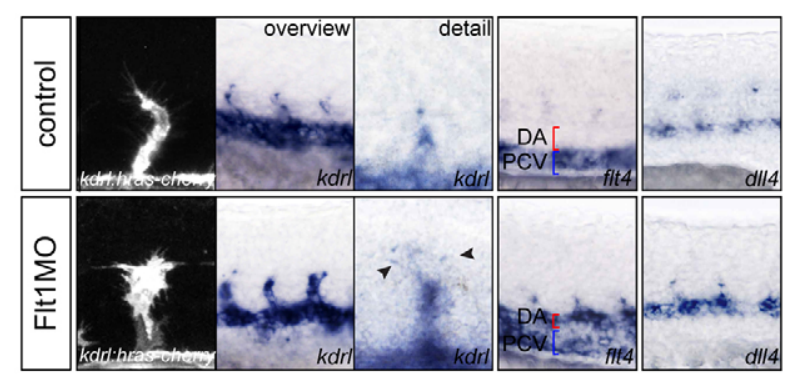

B

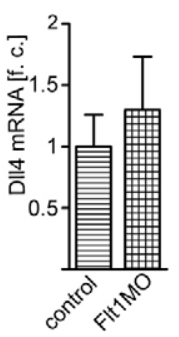

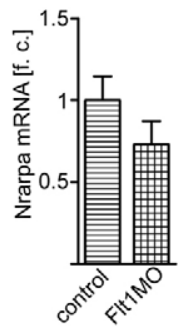

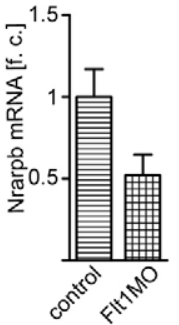

C

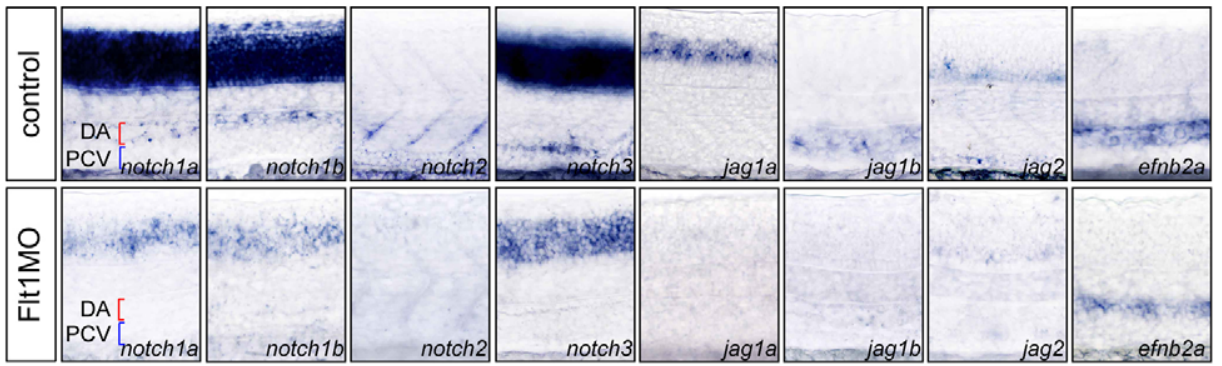

D

E
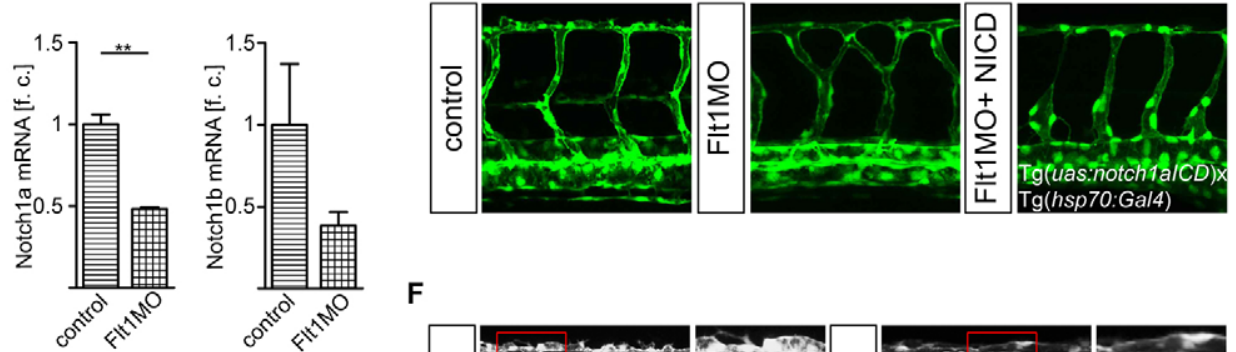

\section{$\mathbf{F}$}
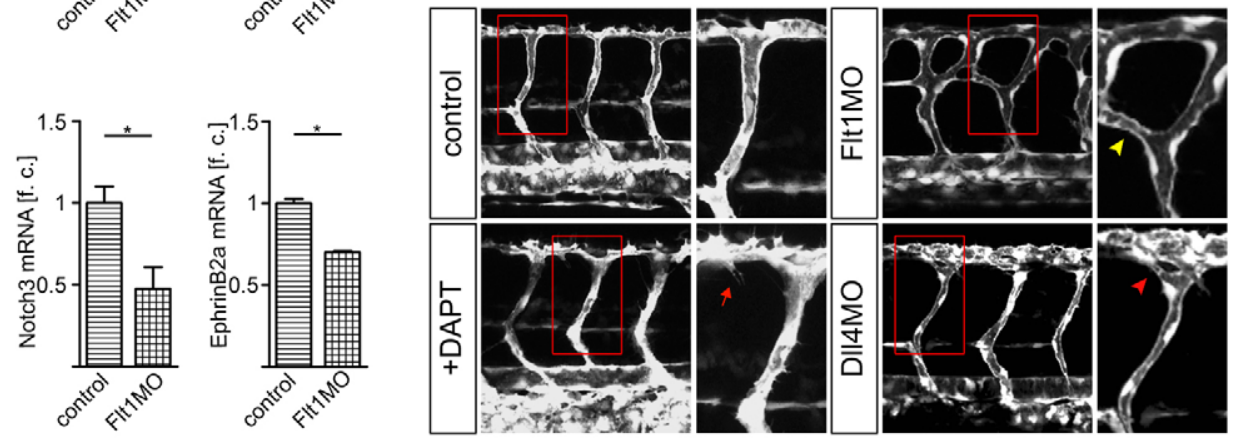

Fig. 4. Tip and stalk cell marker expression and Notch signaling in flt1 morphants. (A,B) Expression of $k d r l$, flt4 and dll 4 in control and flt1 morphants. Expression of the tip cell marker $k d r l$ in segmental sprouts in $T g\left(k d r l\right.$ :hras-mcherry) ${ }^{5896}$ transgenic zebrafish embryos (A, left) and $k d r l$ in toto in situ hybridization (A, overview and detail). Note the ectopic expression of flt4 in the dorsal aorta of flt1 morphants (A, fourth panel). Expression of the stalk cell markers nrarpa and nrarpb is reduced, but expression of $d l l 4$ is maintained in morphants (A, fifth panel; B). (C,D) In situ hybridization and Taqman analysis of the trunk region show reduced expression of the receptors notch1a, notch1b, notch2 and notch3 and of the Notch ligands jag1a, jag $1 \mathrm{~b}$ and jag2 in flt1 morphants. Reduced expression of efnb2a suggests loss of Notch signaling in flt1 morphants. (E) Conditional overexpression of notch1a intracellular cleaved domain (NICD) in flt1 morphants (right) rescues segmental arterial branching defects. (F) Segmental branching pattern in dll4 morphants (red arrowhead) or in embryos treated with the Notch $\gamma$-secretase inhibitor DAPT (red arrow) did not phenocopy the branching pattern of flt1 morphants (yellow arrowhead). The boxed regions are shown at higher magnification in the right-hand panels. DA, dorsal aorta; PCV, posterior cardinal vein. ${ }^{*}, P<0.05 ;{ }^{*}{ }^{*}, P<0.01$; Student's $t$-test. Error bars indicate s.e.m. f.c., fold change relative to age-matched control embryos.

To determine whether the nervous system contains or binds Flt1 protein, we performed immunostaining for Flt1 using a custom-made affinity-purified antibody directed against the extracellular domain of zebrafish Flt1 (Fig. 5C-G; see Fig. S1A and Fig. S4B,C in the supplementary material). The antibody specificity was verified in a series of experiments (see Fig. S2A,B and Fig. S4B,C in the supplementary material). Whole-mount immunostaining for Flt1 in control $\operatorname{Tg}\left(f l t 1^{B A C}: y f p\right)$ or $\operatorname{Tg}(f l i: e g f p)^{y 1}$ embryos showed staining in vessels, neuronal tissue and somites (Fig. 5C-G; see Fig. S4A,B in the supplementary material). 
A
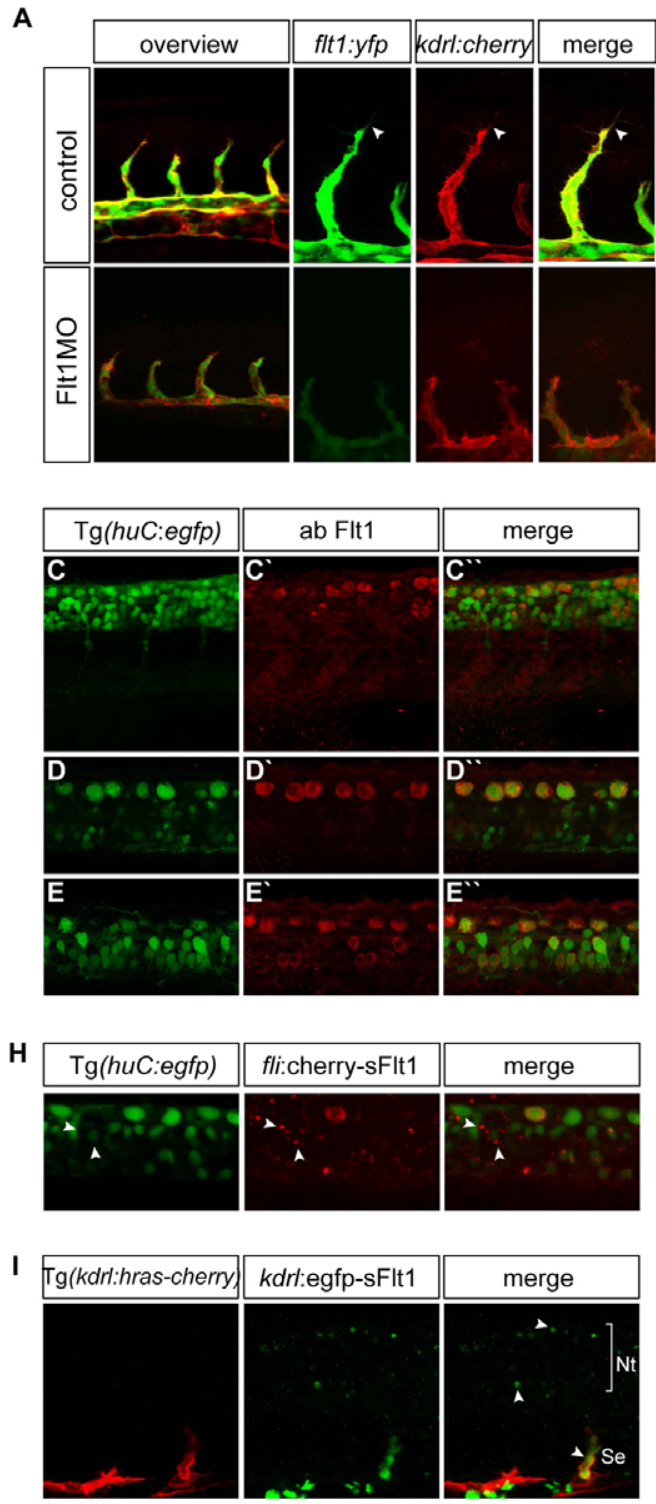

B $\quad \operatorname{Tg}\left(f l t 1^{B A C}: y f p\right) \times \quad \operatorname{Tg}(h u C: e g f p) \times$ $\operatorname{Tg}(k d r l$ :ras-cherry) $\operatorname{Tg}(k d r l$ hras-cherry)
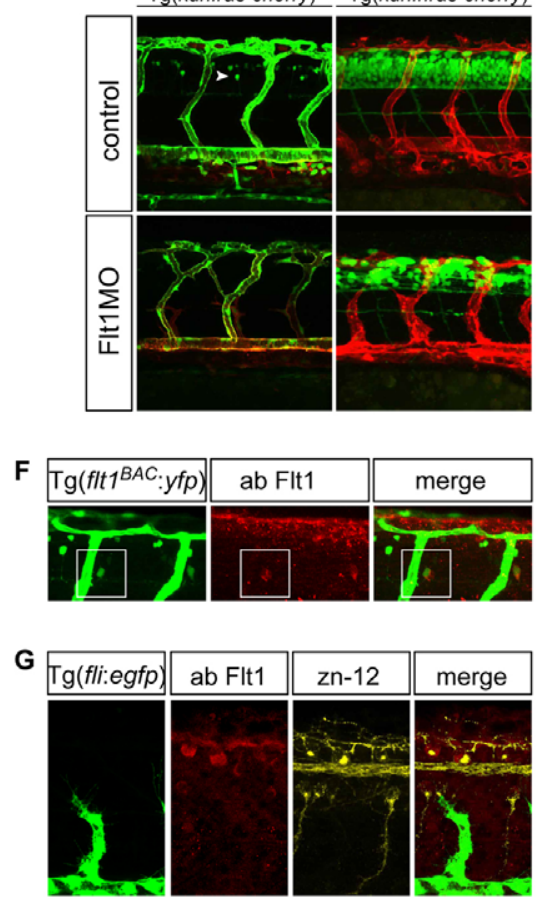

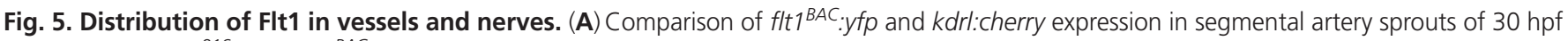
$T g(k d r l: r a s-c h e r r y)^{s 916} \times T g\left(f l t 1^{B A C}: y f p\right)$ double-transgenic zebrafish embryos, in controls (top row) and flt1 morphants (bottom row). In controls, note the overlap of flt $1^{B A C}: y f p$ and $k d r l$ :cherry expression in tip and stalk cells. At $30 \mathrm{hpf}$, expression of flt $1^{\text {BAC }}$ :yfp is restricted to the vascular compartment. After injection of flt1 ATG-blocking MO, expression of $f \mid t t^{B A C}$ :yfp in sprouts appears less intense, but is not completely lost.

(B) Injection of flt1 ATG-blocking MO in Tg(kdrl:ras-cherry $)^{s 916} \times T_{g}\left(f / t 1^{B A C}\right.$ :yfp) double-transgenic embryos causes hyperbranching of segmental vessels and affects neurons. In controls at $48 \mathrm{hpf}$, fIt $1^{B A C}$ :yfp marks segmental vessels and a subset of spinal cord neurons (arrowhead). Injection of the flt1 ATG-blocking MO induces aberrant vessel patterning and the neuronal flt $1^{\text {BAC }}$ :yfp expression domain is reduced. Injection of the flt1 ATGblocking MO in Tg(kdrl:hras-mcherry) ${ }^{5896} \times T g$ (huC:egfp) double-transgenic embryos affects neurons. (C-E") Flt1 immunostaining in Tg(huC:egfp) neuronal reporter embryos shows Flt1 throughout the neural tube. Overview of neural tube (C-C") and different focal planes from dorsal to ventral (D-E") are shown. (F) Flt1 immunostaining in $T g\left(f / t 1^{B A C}\right.$ :yfp) embryos shows co-localization of flt $1^{B A C}$ :yfp with Flt1 antibody staining in spinal cord neurons (boxed). (G) Immunostaining for Flt1 and zn-12 shows Flt1 staining of Rohon-Beard sensory neurons. (H) Overexpression of fli1ep:cherrysflt1 in Tg(huC:egfp) embryos results in neuronal sflt1-cherry expression throughout the neural tube. Arrowheads indicate co-localization of huC:egfp with sFlt1-cherry on neuronal cell bodies. (I) Overexpression of kdrl:egfp-sflt1 in Tg(kdrl:hras-mcherry) ${ }^{5896}$ embryos shows sFlt1-egfp expression in vessels and the neural tube (arrowheads). Nt, neural tube; Se, segmental artery.

$\operatorname{Tg}(h u C: e g f p)$ embryos at 30 hpf showed neuronal Flt1 immunolabeling (Fig. 5C-E), and immunopositive domains included motoneurons, interneurons and sensory nerves. In $\operatorname{Tg}\left(f l t 1^{B A C}: y p f\right)$ embryos, neuronal YPF expression started at $\sim 38$ $40 \mathrm{hpf}$. Immunostaining in $\operatorname{Tg}\left(f l t 1^{B A C}: y p f\right)$ embryos showed colocalization of Flt1 antibody staining with YFP expression in neurons in the spinal cord (Fig. 5F), and it cannot therefore be ruled out that this neuronal population is capable of producing Flt1 protein. However, Flt1 immunostaining was already detectable on neurons at 24-30 hpf, prior to the emergence of flt 1 promoter activity in the nervous system, and not all Flt1 immunopositive neurons expressed YPF. This observation is perhaps best explained by some neurons scavenging soluble Flt1 protein. 
In the period 24-30 hpf, immunostaining showed clear Flt1 localization on cells situated in the dorsal aspect of the trunk (see Fig. S4B in the supplementary material). Based on their position, size and appearance, we speculated that these cells might represent Rohon-Beard primary sensory nerves (Reyes et al., 2004). We performed co-immunostaining for Flt1 and the Rohon-Beard marker zn-12 (Fig. 5G) and confirmed Flt1 immunostaining in Rohon-Beard neurons. flt1 morphants showed reduced Flt1 immunostaining in this domain (see Fig. S4B in the supplementary material). Between 24 and $30 \mathrm{hpf}$, Rohon-Beard neurons lacked flt1 promoter activity (Fig. 5A; first and second upper panels).

We next overexpressed sflt1-cherry under the control of the flilep promoter in $\mathrm{Tg}(\mathrm{huC}$ :egfp) embryos and noted co-localization of sFlt1-cherry with neuronal cell bodies throughout the neural tube (Fig. 5H). In addition, a more diffuse sFlt1-cherry signal was observed in between the neurons (Fig. 5H). Although the fliep promoter may be active in neural crest-derived neural tissue, we clearly observed sFlt1-cherry co-localizing with spinal cord neurons located in the ventral part of the neural tube, and these neurons are not neural crest derived. These observations support the hypothesis that neurons located in the neural tube can bind soluble Flt1 protein produced by other cells. To substantiate that soluble Flt1 produced by the vasculature can reach the neural tube, we overexpressed sflt l-GFP under the control of the $k d r l$ promoter (Fig. 5I) and detected the sFlt1-GFP signal at the level of the neural tube (Fig. 5I, arrowheads).

\section{Flt1 and macrophages}

In mice, Flt1 influences VEGF-induced macrophage migration, and macrophages are implicated in coordinating the fusion of two endothelial tip cells leading to the formation of a vessel anastomosis that can carry blood flow (Fantin et al., 2010). In whole-mount in situ hybridizations for the macrophage marker $l$ plastin (lymphocyte cytosolic plastin 1 - Zebrafish Information Network), we observed reduced l-plastin expression in flt 1 morphants at $30 \mathrm{hpf}$ (see Fig. S3H in the supplementary material). Whereas the brain regions were colonized by macrophages in flt 1 morphants and control embryos, macrophages were less abundant in the trunk region of flt 1 morphants. At $48 \mathrm{hpf}$, l-plastin expression was still slightly reduced in the trunk region of flt 1 morphants (see Fig. S3H,I in the supplementary material). We next inhibited the formation of macrophages by MO knockdown of pul (spil - Zebrafish Information Network) (see Fig. S3J in the supplementary material). Knockdown of pul did not affect sprouting of segmental vessels, neither in the control nor in flt 1 morphants (see Fig. S3J in the supplementary material). It is therefore unlikely that macrophages contribute to the sprouting phenotype of flt 1 morphants.

\section{DISCUSSION}

Here we present Flt1 loss- and gain-of-function data in zebrafish embryos showing that Flt1 regulates tip cell formation and segmental artery branching morphogenesis in a Notch-dependent manner. We furthermore uncovered a role for the nervous system in the distribution of Flt1.

\section{Tip cell formation, Notch signaling and vessel branching in flt1 morphants}

Zebrafish embryos express membrane-bound Flt1 (mFlt1) and soluble Flt1 (sFlt1). In the vasculature, mflt $1 \mathrm{mRNA}$ is expressed in the aorta, the cardinal vein and the segmental arteries, whereas sflt $1 \mathrm{mRNA}$ expression is restricted to the arterial domain. Using
$\operatorname{Tg}\left(f l t 1^{B A C}: y f p\right)$ embryos as a readout, flt1 expression is much stronger in the arterial domain than in the venous domain. In segmental artery sprouts of $\operatorname{Tg}\left(f l t 1^{B A C}: y f p\right)$ embryos, flt1 is expressed in the tip cell, the stalk cell and the base cell, and flt 1 expression overlaps with that of $k d r l$ in these domains.

flt1 morphants show an increase in tip cell number in segmental artery vessel sprouts. In these sprouts, tip cells project filopodia extensions both dorsally and laterally, in anterior and posterior directions, and form connections with sprouts from adjacent segmental vessels. These connections are lumenized, carry blood flow and are not pruned, suggesting that the aberrant branches develop into functional vessels. The extracellular VEGF-binding domain of Flt1 appears sufficient to explain this vascular phenotype, as overexpression of sflt 1 or $m f l t 1$ rescued aberrant branching in flt 1 morphants. In line with this, overexpression of sflt1 or mflt1 resulted in short segmental sprouts and smaller filopodia extensions, most likely by reducing VEGF levels.

In flt1 morphants, we noticed segmental vessel sprouts spearheaded by two endothelial cells that displayed tip cell characteristics, instead of the one tip cell observed in control embryos. Cell tracking revealed that the leading endothelial tip cell gave rise to two progeny cells displaying tip cell behavior. Such tip cell differentiation events have been described in Notch-Dll4 lossof-function models in mouse and zebrafish (Hellstrom et al., 2007; Lobov et al., 2007; Siekmann and Lawson, 2007; Suchting et al., 2007). In addition, endothelial cells displaying tip cell characteristics migrated from the base of the segmental vessel and integrated at the leading, sprouting edge. These movements are highly reminiscent of the endothelial cell movements observed in rbpsuh ( $r$ bpja)-deficient zebrafish embryos (Siekmann and Lawson, 2007). Excessive filopodia activity, increased tip cell numbers and enhanced endothelial cell migration events in sprouts are consistent with loss of Notch signaling (Siekmann and Lawson, 2007). Indeed, flt1 morphants show reduced expression of Notch receptors and of the Notch downstream target efnb2a, as well as ectopic expression of flt 4 in the dorsal aorta, all of which are consistent with loss of Notch signaling in our setting (Siekmann and Lawson, 2007). The expression of dll4 was slightly augmented in flt1 morphants, suggesting intact VEGF receptor signaling, and the loss of Notch signaling in flt1 morphants is thus most likely due to a reduction in Notch expression. Taken together, flt 1 morphants have more tip cells and these tip cells display a high degree of migratory behavior along the developing sprout. We postulate that these two features, combined with a presumed increase in VegfA in the local extravascular compartment caused by loss of soluble Flt1, account for the hyperbranching of segmental arteries.

Notch signaling can regulate angiogenic cell behavior and tip cell differentiation in segmental sprouts (Jakobsson et al., 2010; Siekmann and Lawson, 2007). In a developing sprout, endothelial cells frequently shuffle from the tip to the stalk cell position and vice versa (Jakobsson et al., 2010). Activated Notch signaling restricts such endothelial movements, whereas loss of Notch facilitates shuffling and allows integration of tip cells at the leading edge. Conditional overexpression of NICD rescued segmental vessel patterning defects in flt 1 morphants. In this setting, segmental sprouts reach the dorsal roof and make a DLAV, but endothelial cell movements in the sprout appear to be restricted. Endothelial cells are more frequently localized at the base of the segmental vessel, instead of being distributed evenly along its length. This is compatible with a model in which (conditional) activation of Notch signaling in flt1 morphants limits endothelial cell migration events in the sprout. A reduction in the shuffling of 
endothelial cells (with tip cell characteristics) from the stalk cell/base position towards the leading edge of the segmental sprout might prevent the formation of the additional lateral branches.

Consistent with activated Notch signaling, flt 1 mRNA expression increased after overexpression of NICD, and an increase in Flt1 levels may contribute to the rescue by reducing tip cell formation. Since our data point towards a functional role for the extracellular domain of Flt1 in regulating vascular branching, it remains to be determined how loss of flt 1 causes loss of Notch signaling. In human endothelial cells in vitro, VEGFA stimulates shedding of KDR (VEGFR2), which involves the activation of metalloproteases (Swendeman et al., 2008), but it remains to be clarified to what extent such ectodomain shedding affects $\mathrm{Kdr}$ and Notch signaling at the tip-stalk cell interface in zebrafish.

flt1 morphants show functional and molecular characteristics compatible with a loss of Notch signaling, but knockdown of dll4 or pharmacological inhibition of Notch signaling with DAPT did not phenocopy the vascular branching pattern observed in flt1 morphants. flt1 and dll4 morphants both show ISV branching defects but several differences exist with respect to the onset of hypersprouting (which occurs later in dll4 than in flt1 morphants) and the functionality of the additional sprouts (Leslie et al., 2007). Whereas blood flow through the aorta, cardinal vein and ISVs is severely reduced in dll4 morphants (Leslie et al., 2007), we show that blood flow through these compartments is uncompromised in flt 1 morphants. The lumen diameter of the additional branches in flt 1 morphants is sufficiently large to allow red blood cell perfusion. By contrast, in dll4 morphants, ISVs are largely unperfused, and this difference from $f l t 1$ morphants might involve differential effects on stalk cell differentiation, as these cells are thought to contribute to lumen formation in developing sprouts. Additional differences exist at the level of arterial-venous marker expression. dll4 morphants show unaltered expression of the arterial markers notch 3 and efnb2a and of the venous marker ephb4a (Leslie et al., 2007), whereas flt 1 morphants show reduced expression of Notch receptors and efnb2a and ectopic expression of flt4 in the aorta (at this stage, flt4 is normally expressed in veins and tip cells).

The Dll4-Notch signaling pathway regulates Flt1 expression in stalk cells, but differences might exist in the extent of Flt1 reduction or Flt1 distribution induced by DAPT or dll4 knockdown as compared with flt1 knockdown. In addition, flt1 might exert a functional role beyond the level of the tip-stalk cell interface, independently of Notch. For example, the production of soluble Flt1 by endothelial cells at the base of a developing vessel sprout is relevant for preventing lateral branching or turning of the tip cell back towards the base of the sprout (Chappell et al., 2009). To what extent expression of soluble Flt1 at the base of the sprout is regulated by Notch signaling is not known. We show that the nervous system is a potential source of Flt1. Loss of flt 1 affects neurons, and in flt1 morphants the additional lateral segmental artery branches develop at a level at which the sprout is in close contact with the neural tube. Thus, although both loss of Notch and flt 1 knockdown are associated with reduced Flt1 expression, it is conceivable that differences exist at the level of Flt1 distribution or function that might contribute to the differences in the vascular phenotypes.

\section{Expression and distribution of Flt1 in the developing nervous system}

Flt1 immunostaining shows the presence of Flt1 protein in the developing nervous system of the trunk. We show Flt1 immunostaining in neuronal cells throughout the neural tube, including Rohon-Beard sensory neurons, motoneurons and interneurons. Since, in the trunk, not all of these neurons show flt 1 promoter activity, we postulate that some neuronal populations might actually bind or sequester soluble Flt1 protein produced by other cells.

Examination of the spinal cord in the trunk of $\operatorname{Tg}\left(f l t 1^{B A C}: y f p\right)$ embryos revealed strong YFP expression in interneurons and their axonal projections, and we detected Flt1 protein on interneurons by immunostaining. In rats, retrograde and anterograde transport of VEGF in axons has been demonstrated (Storkebaum et al., 2005), and VEGF appears to be co-transported with its receptor Flk1. Whether mFlt1 or sFlt1 can also be transported along axons remains to be determined. Loss of neuronal YFP expression after knockdown of $f l t 1$ in $\operatorname{Tg}\left(f l t 1^{B A C}: y f p\right)$ embryos might suggest that Flt1 is required for the maintenance or growth of neurons. In mice and rats, the Flt1 ligands VegfA and VegfB promote neuronal survival and exert neuroprotective effects, and reduced expression of these ligands is associated with neurodegenerative diseases (Azzouz et al., 2004; Poesen et al., 2008; Ruiz de Almodovar et al., 2009; Storkebaum et al., 2005). Knockdown of $f l t 1$ in $T g\left(f l t 1^{B A C}: y f p\right)$ embryos predicts increased VegfA and VegfB availability and it is likely that the loss of neurons in our setting is not caused by loss of Flt1 ligands but by reduced $\mathrm{mFlt1}$ expression and signaling. In line with this, VegfB-Flt1 signaling is relevant for the survival of neurons in mice, and in Flt $1^{\text {TK- }-}$ mice, which lack Flt1 signaling, VegfB fails to exert neuroprotective activity (Poesen et al., 2008).

Rohon-Beard cells are mechanosensory neurons which in zebrafish innervate the dorsal caudal fin fold and the skin and play a role in sensing touch (Reyes et al., 2004). Rohon-Beard cells arise around day 1 of development, and during the phase of active segmental vessel remodeling these cells are immunopositive for Flt1, without displaying flt 1 promoter activity. This leaves open the possibility that Rohon-Beard neurons attract or sequester soluble Flt1 protein produced by other cells. It is unclear how these neurons would bind or scavenge soluble Flt1, as soluble Flt1 lacks a membrane-spanning domain. Soluble Flt1 might get to the neurons by diffusion, potentially from sources including the developing trunk vasculature. Since some nerve projections develop in close proximity to segmental vessels, it is also tempting to speculate that developing axons or nerve fibers bind or take up soluble Flt1, which, via retrograde transport, then ends up in the neuronal cell bodies. The existence of such an uptake mechanism remains to be proven. Blood vessels and nerve fibers can often be found alongside one another and, in the mouse embryo skin, the patterning of nerves and blood vessels is interdependent (Mukouyama et al., 2002). Skin sensory nerves determine the pattern of arterial differentiation, which involves the local secretion of VegfA from sensory nerve fibers and Schwann cells (Mukouyama et al., 2002). The open question for future research is whether Flt1 plays a functional role at the neurovascular interface by fine-tuning VEGF levels to determine arterial differentiation and patterning events.

\section{Acknowledgements}

We thank Salim Seyfried for tools and reagents; Didier Stainier for kdrl:rascherry reporter zebrafish; Brant Weinstein, Phillippe Herbomel, Julian Lewis, Marina Mione and Nathan Lawson for plasmids; and Anje Sporbert of the MDC confocal imaging unit for technical support. F.I.N. was supported by grants from the Helmholtz society, ECRC, The Netherlands Organization for Scientific research (NWO) Vidi grant and CSB. This work was also supported by grants from the Medical Research Council (G0600270, G0601295 and G0700288) and British Heart Foundation (RG/09/001/25940). F.I.N. and S.S.-M. are part of the EUFishBioMed COST activity. Deposited in PMC for release after 6 months. 
Competing interests statement

The authors declare no competing financial interests.

\section{Supplementary material}

Supplementary material for this article is available at

http://dev.biologists.org/lookup/suppl/doi:10.1242/dev.063933/-/DC1

\section{References}

Alitalo, K., Tammela, T. and Petrova, T. V. (2005). Lymphangiogenesis in development and human disease. Nature 438, 946-953.

Ambati, B. K., Nozaki, M., Singh, N., Takeda, A., Jani, P. D., Suthar, T. Albuquerque, R. J., Richter, E., Sakurai, E., Newcomb, M. T. et al. (2006) Corneal avascularity is due to soluble VEGF receptor-1. Nature 443, 993-997.

Azzouz, M., Ralph, G. S., Storkebaum, E., Walmsley, L. E., Mitrophanous, K. A., Kingsman, S. M., Carmeliet, P. and Mazarakis, N. D. (2004). VEGF delivery with retrogradely transported lentivector prolongs survival in a mouse ALS model. Nature 429, 413-417.

Benedito, R., Roca, C., Sorensen, I., Adams, S., Gossler, A., Fruttiger, M. and Adams, R. H. (2009). The notch ligands DIl4 and Jagged1 have opposing effects on angiogenesis. Cell 137, 1124-1135.

Bussmann, J., Bakkers, J. and Schulte-Merker, S. (2007). Early endocardial morphogenesis requires Scl/Tal1. PLoS Genet. 3, e140.

Bussmann, J., Lawson, N., Zon, L. and Schulte-Merker, S. (2008). Zebrafish VEGF receptors: a guideline to nomenclature. PLoS Genet. 4, e1000064.

Carmeliet, P., De Smet, F., Loges, S. and Mazzone, M. (2009). Branching morphogenesis and antiangiogenesis candidates: tip cells lead the way. Nat. Rev. Clin. Oncol. 6, 315-326.

Chappell, J. C., Taylor, S. M., Ferrara, N. and Bautch, V. L. (2009). Local guidance of emerging vessel sprouts requires soluble Flt-1. Dev. Cell 17, $377-$ 386

Chi, N. C., Shaw, R. M., De Val, S., Kang, G., Jan, L. Y., Black, B. L. and Stainier, D. Y. (2008). Foxn4 directly regulates tbx2b expression and atrioventricular canal formation. Genes Dev. 22, 734-739.

Covassin, L. D., Villefranc, J. A., Kacergis, M. C., Weinstein, B. M. and Lawson, N. D. (2006). Distinct genetic interactions between multiple Veg receptors are required for development of different blood vessel types in zebrafish. Proc. Natl. Acad. Sci. USA 103, 6554-6559.

Covassin, L. D., Siekmann, A. F., Kacergis, M. C., Laver, E., Moore, J. C., Villefranc, J. A., Weinstein, B. M. and Lawson, N. D. (2009). A genetic screen for vascular mutants in zebrafish reveals dynamic roles for Vegf/Plcg1 signaling during artery development. Dev. Biol. 329, 212-226.

Fantin, A., Vieira, J. M., Gestri, G., Denti, L., Schwarz, Q., Prykhozhij, S., Peri, F., Wilson, S. W. and Ruhrberg, C. (2010). Tissue macrophages act as cellular chaperones for vascular anastomosis downstream of VEGF-mediated endothelial tip cell induction. Blood 116, 829-840.

Ferrara, N., Gerber, H. P. and LeCouter, J. (2003). The biology of VEGF and its receptors. Nat. Med. 9, 669-676.

Fong, G. H., Rossant, J., Gertsenstein, M. and Breitman, M. L. (1995). Role of the Flt-1 receptor tyrosine kinase in regulating the assembly of vascular endothelium. Nature 376, 66-70

Gerhardt, H., Golding, M., Fruttiger, M., Ruhrberg, C., Lundkvist, A. Abramsson, A., Jeltsch, M., Mitchell, C., Alitalo, K., Shima, D. et al. (2003). VEGF guides angiogenic sprouting utilizing endothelial tip cell filopodia. J. Cell Biol. 161, 1163-1177.

Habeck, H., Odenthal, J., Walderich, B., Maischein, H. and Schulte-Merker, S. (2002). Analysis of a zebrafish VEGF receptor mutant reveals specific disruption of angiogenesis. Curr. Biol. 12, 1405-1412.

Hellstrom, M., Phng, L. K., Hofmann, J. J., Wallgard, E., Coultas, L., Lindblom, P., Alva, J., Nilsson, A. K., Karlsson, L., Gaiano, N. et al. (2007). Dll4 signalling through Notch1 regulates formation of tip cells during angiogenesis. Nature $\mathbf{4 4 5}, \mathbf{7 7 6 - 7 8 0}$

Hiratsuka, S., Minowa, O., Kuno, J., Noda, T. and Shibuya, M. (1998). Flt-1 lacking the tyrosine kinase domain is sufficient for normal development and angiogenesis in mice. Proc. Natl. Acad. Sci. USA 95, 9349-9354.

Hogan, B. M., Bos, F. L., Bussmann, J., Witte, M., Chi, N. C., Duckers, H. J. and Schulte-Merker, S. (2009a). Ccbe1 is required for embryonic lymphangiogenesis and venous sprouting. Nat. Genet. 41, 396-398.

Hogan, B. M., Herpers, R., Witte, M., Helotera, H., Alitalo, K., Duckers, H. J. and Schulte-Merker, S. (2009b). Vegfc/Flt4 signalling is suppressed by Dll4 in developing zebrafish intersegmental arteries. Development 136, 4001-4009.

Hsu, K., Traver, D., Kutok, J. L., Hagen, A., Liu, T. X., Paw, B. H., Rhodes, J., Berman, J. N., Zon, L. I., Kanki, J. P. et al. (2004). The pu.1 promoter drives myeloid gene expression in zebrafish. Blood 104, 1291-1297.

Isogai, S., Lawson, N. D., Torrealday, S., Horiguchi, M. and Weinstein, B. M. (2003). Angiogenic network formation in the developing vertebrate trunk. Development 130, 5281-5290

Jakobsson, L., Franco, C. A., Bentley, K., Collins, R. T., Ponsioen, B., Aspalter, I. M., Rosewell, I., Busse, M., Thurston, G., Medvinsky, A. et al. (2010).
Endothelial cells dynamically compete for the tip cell position during angiogenic sprouting. Nat. Cell Biol. 12, 943-953.

Jowett, T. and Lettice, L. (1994). Whole-mount in situ hybridizations on zebrafish embryos using a mixture of digoxigenin- and fluorescein-labelled probes. Trends Genet. 10, 73-74

Lawson, N. D. and Weinstein, B. M. (2002). In vivo imaging of embryonic vascular development using transgenic zebrafish. Dev. Biol. 248, 307-318.

Lawson, N. D., Vogel, A. M. and Weinstein, B. M. (2002). sonic hedgehog and vascular endothelial growth factor act upstream of the Notch pathway during arterial endothelial differentiation. Dev. Cell 3, 127-136.

Leslie, J. D., Ariza-McNaughton, L., Bermange, A. L., McAdow, R., Johnson, S. L. and Lewis, J. (2007). Endothelial signalling by the Notch ligand Delta-like 4 restricts angiogenesis. Development 134, 839-844.

Liao, W., Bisgrove, B. W., Sawyer, H., Hug, B., Bell, B., Peters, K., Grunwald, D. J. and Stainier, D. Y. (1997). The zebrafish gene cloche acts upstream of a flk-1 homologue to regulate endothelial cell differentiation. Development 124 381-389.

Lobov, I. B., Renard, R. A., Papadopoulos, N., Gale, N. W., Thurston, G., Yancopoulos, G. D. and Wiegand, S. J. (2007). Delta-like ligand 4 (D\|4) is induced by VEGF as a negative regulator of angiogenic sprouting. Proc. Natl. Acad. Sci. USA 104, 3219-3224.

Mukouyama, Y. S., Shin, D., Britsch, S., Taniguchi, M. and Anderson, D. J. (2002). Sensory nerves determine the pattern of arterial differentiation and blood vessel branching in the skin. Cell 109, 693-705

Park, H. C., Kim, C. H., Bae, Y. K., Yeo, S. Y., Kim, S. H., Hong, S. K., Shin, J., Yoo, K. W., Hibi, M., Hirano, T. et al. (2000). Analysis of upstream elements in the HuC promoter leads to the establishment of transgenic zebrafish with fluorescent neurons. Dev. Biol. 227, 279-293.

Phng, L. K. and Gerhardt, H. (2009). Angiogenesis: a team effort coordinated by notch. Dev. Cell 16, 196-208.

Phng, L. K., Potente, M., Leslie, J. D., Babbage, J., Nyqvist, D., Lobov, I. Ondr, J. K., Rao, S., Lang, R. A., Thurston, G. et al. (2009). Nrarp coordinates endothelial Notch and Wnt signaling to control vessel density in angiogenesis. Dev. Cell 16, 70-82.

Poesen, K., Lambrechts, D., Van Damme, P., Dhondt, J., Bender, F., Frank, N., Bogaert, E., Claes, B., Heylen, L., Verheyen, A. et al. (2008). Novel role for vascular endothelial growth factor (VEGF) receptor-1 and its ligand VEGF-B in motor neuron degeneration. J. Neurosci. 28, 10451-10459.

Reyes, R., Haendel, M., Grant, D., Melancon, E. and Eisen, J. S. (2004). Slow degeneration of zebrafish Rohon-Beard neurons during programmed cell death. Dev. Dyn. 229, 30-41.

Roberts, D. M., Kearney, J. B., Johnson, J. H., Rosenberg, M. P., Kumar, R. and Bautch, V. L. (2004). The vascular endothelial growth factor (VEGF) receptor Flt-1 (VEGFR-1) modulates Flk-1 (VEGFR-2) signaling during blood vessel formation. Am. J. Pathol. 164, 1531-1535.

Roca, C. and Adams, R. H. (2007). Regulation of vascular morphogenesis by Notch signaling. Genes Dev. 21, 2511-2524.

Rottbauer, W., Just, S., Wessels, G., Trano, N., Most, P., Katus, H. A. and Fishman, M. C. (2005). VEGF-PLCgamma1 pathway controls cardiac contractility in the embryonic heart. Genes Dev. 19, 1624-1634.

Ruiz de Almodovar, C., Lambrechts, D., Mazzone, M. and Carmeliet, P. (2009). Role and therapeutic potential of VEGF in the nervous system. Physiol. Rev. 89, 607-648.

Sela, S., Itin, A., Natanson-Yaron, S., Greenfield, C., Goldman-Wohl, D. Yagel, S. and Keshet, E. (2008). A novel human-specific soluble vascular endothelial growth factor receptor 1, cell-type-specific splicing and implications to vascular endothelial growth factor homeostasis and preeclampsia. Circ. Res. 102, 1566-1574

Siekmann, A. F. and Lawson, N. D. (2007). Notch signalling limits angiogenic cell behaviour in developing zebrafish arteries. Nature 445, 781-784.

Storkebaum, E., Lambrechts, D., Dewerchin, M., Moreno-Murciano, M. P., Appelmans, S., Oh, H., Van Damme, P., Rutten, B., Man, W. Y., De Mol, M. et al. (2005). Treatment of motoneuron degeneration by intracerebroventricular delivery of VEGF in a rat model of ALS. Nat. Neurosci. 8, 85-92.

Suchting, S., Freitas, C., le Noble, F., Benedito, R., Breant, C., Duarte, A. and Eichmann, A. (2007). The Notch ligand Delta-like 4 negatively regulates endothelial tip cell formation and vessel branching. Proc. Natl. Acad. Sci. USA 104, 3225-3230

Swendeman, S., Mendelson, K., Weskamp, G., Horiuchi, K., Deutsch, U., Scherle, P., Hooper, A., Rafii, S. and Blobel, C. P. (2008). VEGF-A stimulates ADAM17-dependent shedding of VEGFR2 and crosstalk between VEGFR2 and ERK signaling. Circ. Res. 103, 916-918.

Tammela, T., Zarkada, G., Wallgard, E., Murtomaki, A., Suchting, S., Wirzenius, M., Waltari, M., Hellstrom, M., Schomber, T., Peltonen, R. et al. (2008). Blocking VEGFR-3 suppresses angiogenic sprouting and vascular network formation. Nature 454, 656-660

Villefranc, J. A., Amigo, J. and Lawson, N. D. (2007). Gateway compatible vectors for analysis of gene function in the zebrafish. Dev. Dyn. 236, 3077-3087. 